\title{
Parallelization of Generic Libraries Based on Type Properties
}

\author{
Prabhanjan Kambadur, Douglas Gregor, and Andrew Lumsdaine \\ Open Systems Laboratory \\ Indiana University \\ Bloomington, IN 47405 \\ \{pkambadu, dgregor, lums\}@osl.iu.edu
}

\begin{abstract}
This paper describes a new approach for parallelizing generic software libraries. Generic algorithms are expressed in terms of type properties, which allows them to work with entire families of types rather than specific types. Despite this generality, generic algorithms can be made as efficient as their hand-coded variants through the use of specialization, which provides algorithm variants tuned for types with certain properties. Our approach leverages the specialization mechanism of generic programming to effect parallelism. We illustrate the process of specializing generic algorithms for parallelism using common algorithms in the C++ Standard Template Library. When the resulting algorithms are invoked with types that have the required properties for parallelization, the parallel variants of these algorithms are executed. We illustrate that our parallelization strategy is simple, practical, and efficient, using tools and techniques available in most commercial compilers.
\end{abstract}

Keywords: Parallel Algorithms, Specialization, Type Properties.

\section{Introduction}

The advent of many-core and multi-core processors will significantly broaden the applicability of parallel computing. While this new era of ubiquitous parallelism will surely bring new languages and tools built from the ground up for parallelism, a vast majority of tomorrow's potential parallel applications are today's sequential programs. The primary challenge to ubiquitous parallelism is the need to ease the transition from sequential to parallel, allowing existing applications to be parallelized without requiring them to be rewritten or refactored.

The ultimate goal for enabling parallelization of sequential applications is the fully automatic parallelizing compiler. Although there has been research on such compilers for many years, practical solutions have remained elusive. An alternate solution to parallelization is to provide user-level libraries to aid in writing explicitly parallel applications. Various user-level libraries such as pthreads and MPI have provided services that enable parallelization of applications. However, the programming interface that these libraries offer is very low-level. Thus, they are both difficult to use and often lack support for the abstractions of relatively high-level languages like $\mathrm{C}++$. 
A first step towards parallelizing existing sequential applications is to parallelize those components (libraries) that are most commonly used by these applications. When parallel versions of commonly used libraries become available, previously sequential applications are "automatically" parallelized. This approach has been successfully followed in many scientific libraries (for example, BLAS [1] 2]). In this paper, we describe the methodology by which we are able to accurately parallelize applications that use generic libraries. For example, we are able to parallelize the following code segment:

std::vector $<$ int $>\operatorname{vec}(100)$;

// Initialize vec...

std::accumulate (vec.begin(), vec.end(), 1, std::multiplies $<$ int $>())$;

This code segment calls accumulate(), a generic algorithm implemented in the C++ Standard Template Library (STL) 3]. The STL is an excellent example of Generic Programming, an important programming paradigm that is used for the development of highly efficient and maximally reusable software 4 . Moreover, the STL is a widely-used portion of the C++ Standard Library, so parallelizing it can have an immediate impact on many existing $\mathrm{C}++$ applications.

In this paper, we illustrate the parallelization of the generic algorithms in the STL through the use of type properties, and demonstrate how our approach automatically parallelizes calls to STL algorithms. Our parallelization is based on OpenMP, a set of compiler directives and library routines that enable users to express shared-memory parallelism. We choose OpenMP because it is simple, intuitive to use and is available in most modern $\mathrm{C}++$ compilers. This last point is particularly important: OpenMP, C++, and the STL are all existing, widelydeployed and widely-used technologies. By building on these technologies, our OpenMP-parallel implementation of the STL can be used to parallelize existing applications without requiring developers or end-users to install new tools or runtime systems; merely recompiling with our OpenMP-parallel STL is sufficient.

\section{Related Work}

ROSE [5] is a C++ infrastructure for source-to-source translation that provides an interface for writing customized translators that optimize user-defined high-level abstractions. One such translator automatically inserts OpenMP statements into user code. In principle, ROSE is akin to what we intend to achieve. However, the methodologies are vastly different. ROSE is an external tool that all developers must install whereas we advocate a minimal approach based only on $\mathrm{C}++$ and OpenMP, both of which are widely available.

STAPL 6] [7] is designed in much the same spirit as the STL with containers, iterators and algorithms. STAPL also allows users to specify their own scheduling policies, data composition and data dependence environment. STAPL is designed to operate on both shared and distributed memory systems and, therefore, it includes an advanced runtime system. Existing applications need to be rewritten to benefit from STAPL, and end-users must install the STAPL runtime system to use these applications. 
$\mathrm{HPC}++[8$ is a C++ class and template library that portably supports sharedmemory and distributed-memory parallel applications. HPC++ supports both task and data parallel applications. Unlike our approach, HPC++ delivers parallelism through a Java-like class library and consequently suffers from many of the same drawbacks as STAPL. HPC++ is no longer in active development.

Threading Building Blocks (TBB) [?] is a library-based solution to sharedmemory parallelism that is tailored to the high-level of $\mathrm{C}++$ abstractions. TBB offers a wide variety of customizable parallel algorithms and concurrent datastructures that facilitate development of parallel applications. However, parallelizing applications with TBB encumbers some amount of rewriting.

\section{Generic Programming}

Most modern software libraries consist of collections of related functions and data types, often as archives of object files in conjunction with header files that define interfaces to components in the library. Although the goal of these libraries is to promote reuse, the requirements for invoking functions in these libraries are often over-specified. This leads to a less than optimal reuse of the components.

With the explicit goal of improving the reuse of software libraries, generic programming has emerged as an important paradigm for the development of highly efficient and maximally reusable software 4. Generic programming involves the implementation of generic (abstract) algorithms that operate correctly and efficiently on a wide range of data types - including those not known at algorithm design time - so long as the data types meet certain requirements expressed as type properties. Related type properties are grouped together into concepts, which identify domain-specific abstractions such as a Matrix, Vector, Graph, or Iterator on which generic algorithms will operate.

$\mathrm{C}++$ does not provide an inherent way to specify concepts and, therefore, there exists no direct means of checking if a type models a particular concept. However, we can approximate this behavior through the use of traits classes. Traits are a $\mathrm{C}++$ template technique that allow one to provide additional information about a set of types without modifying the types themselves [9. A key advantage of using traits classes is that type properties can be attached retroactively. This enables attachment of properties to types whose definitions are not available.

In many cases, we can improve the performance of a generic algorithm by exploiting additional type properties. For example, consider a generic algorithm that computes the circumference of an arbitrary polygon: it will require linear time to sum the edge lengths. However, if we restrict the algorithm to equilateral polygons, we can compute the circumference in constant time. A generic library typically provides the most generic algorithm (e.g., for arbitrary polygons) along with several specializations of the algorithm (e.g., for equilateral polygons). When a user invokes the generic algorithm, the library will automatically select the most specialized algorithm at compile time, ensuring that the library provides the best possible performance. In practice "selecting" the right 


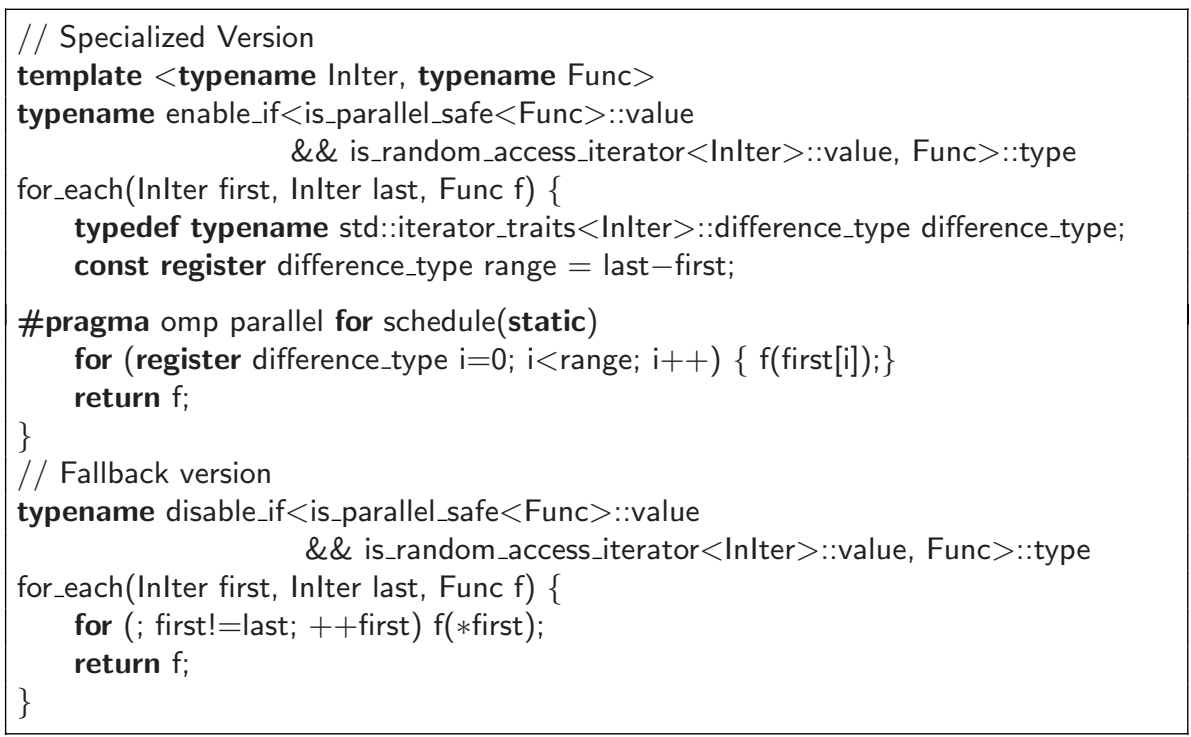

Fig. 1. Parallel implementation of the STL for_each() algorithm

algorithm often involves accessing traits classes to ensure that the input types to the specialized algorithm meet all the type requirements.

\section{Parallelizing Libraries}

Specializations based on type properties can be used to extract optimal performance out of generic algorithms. Type properties serve as gate keepers that ensure that input types meet the requirements stipulated by specialized versions of algorithms. In this section, we elaborate through examples, the process of parallelizing generic algorithms based on type properties.

First, consider the STL algorithm for_each(), which applies a unary function to all the values in the range [first, last).

template <typename Inlter, typename Func $>$

Func for_each (Inlter first, Inlter last, Func f);

This algorithm stipulates that Inlter model the Inputlterator concept, Func model the UnaryFunction concept (i.e., have operator ( ) defined that accepts one argument and returns a result) and that Inlter's value type be convertible to the argument type of Func. Although these type requirements are necessary and sufficient for sequential correctness, to parallelize for_each() using OpenMP, input types must possess additional properties. As OpenMP can only parallelize for loops that are expressed in a simple single induction variable form, Inlter must model the RandomAccesslterator concept. Similarly, Func has to be reentrant and give the same results regardless of the order in which the values are supplied to 
it, i.e., Func must model the ParallelSafe concept. This particular requirement ensures that it is safe to execute Func in parallel.

To check for these two additional type properties (i.e., meeting the ParallelSafe and RandomAccesslterator concept requirements), we make use of the metafunctions is_parallel_safe and is_random_access_iterator. is_parallel_safe is a simple templated structure that by default specifies that no function object is safe to be executed in parallel. To specify that the type plugged into Func models the ParallelSafe concept, users need to fully specialize the is_parallel_safe structure. This is nothing but a means of specifying traits for function objects. For convenience, is_parallel_safe has already been specialized for all the STL function objects. Similarly, is_random_access_iterator is used to determine whether a particular iterator models the RandomAccesslterator concept. Like is_parallel_safe, is_random_access_iterator is pre-defined for all the STL iterators. The metafunctions enable_if and disable_if [10] are used to ensure that only one of the versions of for_each() is "turned on" when instantiated with concrete types. The code fragment that implements this specialization is given in Figure 1. To demonstrate when the parallel for_each is invoked, consider the following example.

struct square_f $\{$ void operator ()$($ int $\&$ value $)\{$ value $*=$ value; $\}$;

template $<>$ struct is_parallel_safe $<$ square_f $>\{$ static const bool value $=$ true; $\}$;

std::vector $<$ int $>\operatorname{vec}(100)$;

std: :list $<$ int $>$ lst $(100)$;

for_each(vec.begin(), vec.end(), square_f());// PARALLEL

for_each(Ist.begin(), Ist.end(), square_f());// SEQUENTIAL

The first call to for_each() is supplied iterators to std::vector and a function object of type square_f. Since std::vector iterators support random access and square_f is safely invokable in parallel (as specified through the specialization of the is_parallel_safe structure), this call is parallelized. The second call to for_each() uses the same function object as the first, but is instantiated with iterators to std::list, which do not support random access. Consequently, this call is not parallelized and is executed sequentially instead.

Consider accumulate(), an STL algorithm that reduces based on some reduction operator, all the values in the iterator range [first,last). For example, the code fragment given below computes the sum of all the elements in the array.

int array []$=\{1,2,3,4\}$;

std::cout $<<$ accumulate $(a, a+4,10$, std::plus $<$ int $>())$;

This fragment outputs 20, which is the sum of all the elements of the array plus the initial value of 10 . The prototype of accumulate() is given below.

template <typename Inlter, typename $\mathrm{T}$, typename Func $>$

$\mathrm{T}$ accumulate (Inlter first, Inlter last, $\mathrm{T}$ init, Func binary_op);

The type requirements for accumulate() stipulate that Inlter model the Inputlterator concept, $T$ be Assignable, $T$ be convertible to value type of InIter and Func model the BinaryFunction concept (i.e, have operator () defined that accepts two arguments and returns a result). As in the case of for_each(), to parallelize this algorithm using 


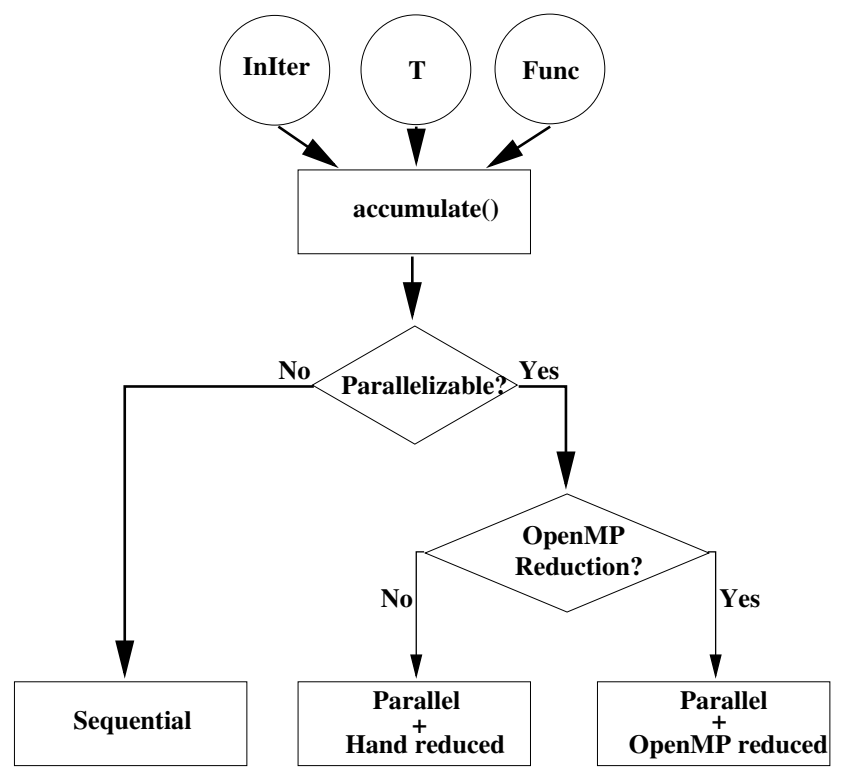

Fig. 2. Decision Logic followed for accumulate()

OpenMP, Inlter is required to model the RandomAccesslterator concept and Func to model the ParallelSafe concept. However, if we also want to use OpenMP's reduction clause, we require $T$ to be a fundamental type such as an int and Func to be one of $\{+,-, * \&, \mid, \wedge \& \&, \|\}$ operators.

Therefore, we can parallelize accumulate() at two levels. First, we decide on whether the input types meet the basic criteria for parallelization. If they do meet these requirements, we then decide if we can use OpenMP's reduction clause. If it cannot be used, we manually reduce the results from the various threads at the very end. Thus, we provide two different parallel specializations of the accumulate() algorithm, the most suitable of which will be chosen at compile time. Had we provided only one of these, we would have either limited the parallelism (e.g., by only providing the more limited OpenMP reduction) or limited the potential for better performance (e.g., by using a manually-coded reduction where an architecture-optimized OpenMP reduction would be better).

The decision process for accumulate() is illustrated by means of a flowchart in Figure 2. To illustrate the decision logic, we give some of code examples and explain which final version of accumulate() is used. First, consider the following code that uses some STL iterators and STL function objects.

std::vector $<$ int $>$ array $(100) ; \ldots$

int sum $=$ accumulate $($ array.begin(), array.end(), 1, std::multiplies $<$ int $>())$;

Here, iterator to std::vector is plugged in for Inlter, int for $\mathrm{T}$ and std::multiplies $<>$ for Func. As iterators to std::vector model RandomAccesslterator concept, int is a fundamental type and $\mathbf{s t d}:$ :multiplies $<$ int $>$ can be mapped to the primitive 


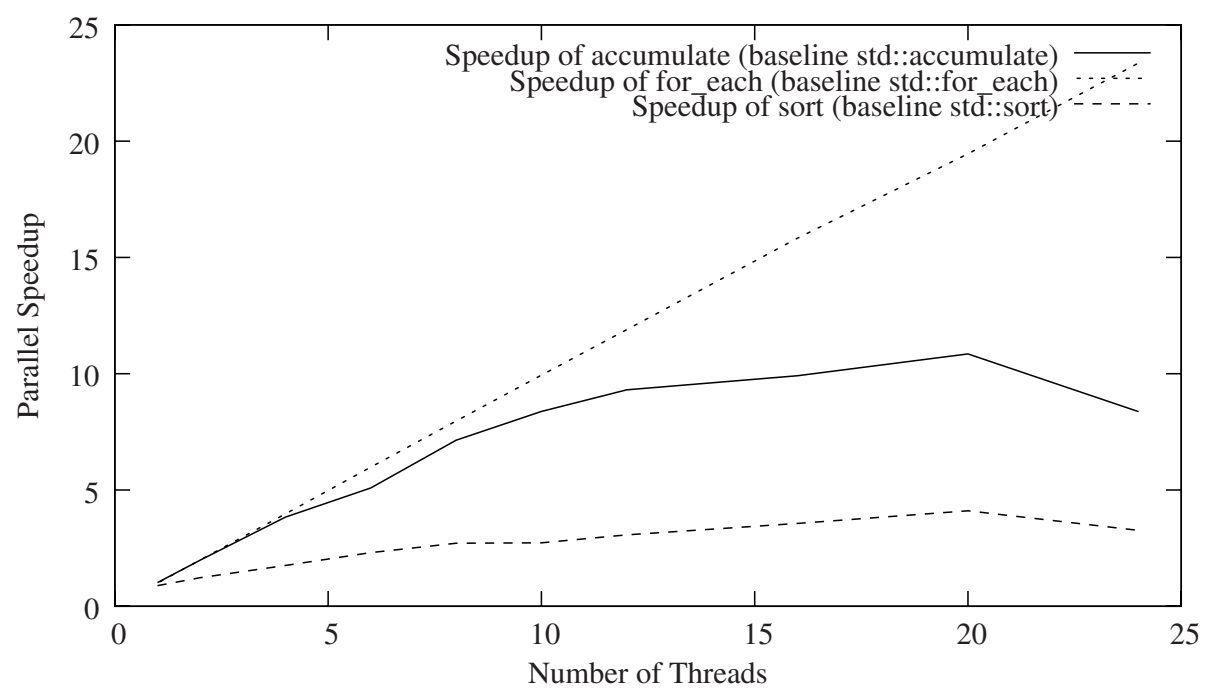

Fig. 3. Parallel speedup achieved for several STL algorithms on a 32-way SMP

* operator, the parallel version with OpenMP reduction is used. Consider the following example that uses fundamental types and user-defined function objects.

std::vector $<$ int $>$ array $(100) ; \ldots$

int sum $=$ accumulate $($ array.begin () , array.end ()$, 0$, my_bin_op ()$)$;

Although fundamental types are used, the iterators support random access, and calls to my_bin_op can be parallelized (assuming is_parallel_safe is specialized), OpenMP reductions cannot be applied because there is no primitive operator to which we can map my_bin_op. Instead, we parallelize this invocation using a hand-coded reduction. The same logic applies if $\mathrm{T}$ were a non-fundamental type and a "known" function object such as std::plus $<>$ were used. Furthermore, if, in this example, array were to be a std::list instead of a std::vector, no parallelization would be possible since iterators to std::list do not support random access.

\section{Performance Evaluation}

We have developed parallel specializations for several algorithms in the STL. In this section, we give performance results for three of the algorithms: for_each(), sort() and accumulate(). Tests were run on a 32-way IBM AIX p690 SMP using 1.3 GHz Power4 processors with 192 GB of memory. IBM's LoadLeveler was used for batch queuing. XLC compiler version 7.0 was used with the - qsmp and -O3 flags. The performance results are given in Figure 3. All results are for 1 million randomly generated floating point numbers that were stored in a std::vector. sort () showed a maximum speedup of around $4 \mathrm{x}$ at 20 threads, accumulate() showed a speedup of $11 \mathrm{x}$ for 20 threads. for_each(), which is an embarassingly parallel algorithm, scales linearly with the number of threads for this particular problem size. 


\section{Conclusions}

In this paper, we have presented a new methodology for parallelizing generic libraries based on type properties. Using modern $\mathrm{C}++$ idioms such as specialization and traits, we can isolate candidate invocations of generic algorithms for automatic parallelization at the library level. Unlike most approaches to automatic parallelization or library-level parallelization, which require new compilers, source-tosource translation tools, or new run-time systems, our solution works with existing, widely-deployed technologies (OpenMP and C++).

Preliminary results show that this approach gives excellent parallel speedup for commonly used algorithms such as accumulate(), sort() and for_each() on large shared-memory machines; we have seen similar results on commodity desktops and servers as well.

Acknowledgements. This work was supported by NSF grants EIA-0202048, EIA-0131354, a grant by the Lilly Endowment and in part by Shared University Research grants from IBM, Inc. to Indiana University.

\section{References}

1. Alpatov, P., Baker, G., Edwards, C., Gunnels, J., Morrow, G., Overfelt, J., van de Geijn, R.: PLAPACK: Parallel linear algebra package. In: SIAM Parallel Processing Conference. (1997)

2. Blackford, L.S., Choi, J., Cleary, A., D'Azevedo, E., Demmel, J., Dhillon, I., Dongarra, J., Hammarling, S., Henry, G., Petitet, A., Stanley, K., Walker, D., Whaley, R.C.: ScaLAPACK: a linear algebra library for message-passing computers. In: Proceedings of the Eighth SIAM Conference on Parallel Processing for Scientific Computing (Minneapolis, MN, 1997). (1997)

3. Stepanov, A.A., Lee, M.: The Standard Template Library. Technical Report X3J16/94-0095, WG21/N0482, ISO Programming Language C++ Project (1994)

4. Musser, D.R., Stepanov, A.A.: Generic programming. In Gianni, P.P., ed.: Symbolic and algebraic computation: ISSAC '88, Rome, Italy, July 4-8, 1988: Proceedings. Volume 358 of Lecture Notes in Computer Science., Berlin, Springer Verlag (1989) $13-25$

5. Quinlan, D.: ROSE: Compiler support for object-oriented frameworks. Parallel Processing Letters 10(2,3) (2000) 215-226

6. An, P., Jula, A., Rus, S., Saunders, S., Smith, T., Tanase, G., Thomas, N., Amato, N., Rauchwerger, L.: STAPL: A standard template adaptive parallel C++ library. In: Int. Wkshp on Adv. Compiler Technology for High Perf. and Embedded Processors. (2001) 10

7. An, P., Jula, A., Rus, S., Saunders, S., Smith, T., Tanase, G., Thomas, N., Amato, N., Rauchwerger, L.: STAPL: An adaptive, generic parallel programming library for C++. In: Wkshp. on Lang. and Comp. for Par. Comp. (LCPC). (2001) 193-208

8. Gannon, D., Beckman, P., Johnson, E., Green, T., Levine, M.: HPC++ and the $\mathrm{HPC}++$ Lib Toolkit. (The High Performance $\mathrm{C}++$ consortium)

9. Myers, N.C.: Traits: a new and useful template technique. C++ Report (1995)

10. Järvi, J., Willcock, J., Hinnant, H., Lumsdaine, A.: Function overloading based on arbitrary properties of types. C/C++ Users Journal 21(6) (2003) 25-32 